\title{
GTI-2501, an antisense agent targeting $R 1$, the large subunit of human ribonucleotide reductase, shows potent anti-tumor activity against a variety of tumors
}

\author{
YOON LEE, AIKATERINI VASSILAKOS, NINGPING FENG, HONGNAN JIN, MING WANG, \\ KEYONG XIONG, JIM WRIGHT and AIPING YOUNG
}

Lorus Therapeutics Inc., 2 Meridian Road, Ontario, M9W 4Z7, Canada

Received October 6, 2005; Accepted November 29, 2005

\begin{abstract}
GTI-2501 is a 20-mer oligonucleotide that is complementary to a coding region in the mRNA of R1, the large subunit of ribonucleotide reductase (RNR). In vitro studies, have demonstrated that GTI-2501 decreases mRNA and protein levels of R1 in a sequence-specific and dosedependent manner. Furthermore, GTI-2501 inhibits the growth of human lung, liver, ovary, brain, melanoma, breast and pancreatic tumor cells in colony forming assays. In vivo studies have shown that GTI-2501 significantly inhibits growth of human colon, pancreas, lung, breast, renal, ovarian, melanoma, brain glioblastoma-astrocytoma, and prostatic tumors in CD-1 nude, Balb/c nude and/or SCID mice. GTI-2501 treatment caused total regression of human breast and renal tumor xenografts in mice. These effects are not observed with a scrambled control oligonucleotide containing the same base content but not complementary to R1. GTI-2501 specifically inhibits metastasis of human melanoma cells to the lungs in CD-1 athymic nude mice and prolongs the survival of mice bearing human lymphoma. Taken together these results suggest that an antisense mechanism of action is responsible for growth inhibition in vitro and in vivo and that GTI-2501 can act as a selective and specific anti-tumor agent.
\end{abstract}

Correspondence to: Dr Yoon Lee, Lorus Therapeutics Inc., 2 Meridian Road, Ontario, M9W 4Z7, Canada

E-mail: ylee@lorusthera.com

Abbreviations: RNR, ribonucleotide reductase; ODN, oligodeoxynucleotide; AS-ODN, antisense oligodeoxynucleotide; PS-ASODN, phosphorothioate antisense oligodeoxynucleotide; 5' UTR, 5' untranslated region; 3' UTR, 3' untranslated region; SCID, severe combined immune deficient; kDa, kilodalton; NK, natural killer; HU, hydroxyurea; 5-FU, 5-fluorouracil; dNTP, deoxynucleoside triphosphate; FBS, fetal bovine serum; FCS, fetal calf serum; RB, retinoblastoma; GAPDH, glyceraldehyde-3-phosphate dehydrogenase; $\alpha$-MEM, minimal essential medium; HUVEC, human umbilical vein endothelial cells; MAPK, mitogen-activated protein kinase-2; PBS, phosphate-buffered saline; HRP, horseradish peroxidase

Key words: ribonucleotide reductase, antisense, oligonucleotides, anti-tumor, therapeutic

\section{Introduction}

In 2005 , it is expected that $1,372,910$ new cases of invasive cancer will be diagnosed in the United States and 570,280 people are expected to die from cancer (American Cancer Society, Facts and Figures 2005). Current therapeutic approaches to cancer include surgery, radiation, chemotherapy, hormone and cytokine therapy. Each of these therapies has limited efficacy and can result in toxicity to normal cells. Thus, there is a need for therapies that specifically target tumor cells thereby having more favorable safety profiles. Several therapeutic agents are currently used which inhibit ribonucleotide reductase (RNR) as part of their mechanism of action. These include hydroxyurea (Hydrea ${ }^{\circledR}$, Bristol-Myers Squibb and Hydroxyurea Capsules, Roxane), gemcitabine $\left(\right.$ Gemzar $^{\circledR}$, Eli Lilly) and fludarabine (Fludara ${ }^{\circledR}$, Berlex). Gemcitabine and fludarabine are not specific inhibitors of ribonucleotide reductase and treatment results in significant side effects that limit their effectiveness. Hydroxyurea is a reversible inhibitor of RNR that requires relatively high concentrations to be effective.

Ribonucleotide reductase catalyzes the reaction in which 2'-deoxyribonucleotides (dADP, dGDP, dUDP, and dCDP) are synthesized from the corresponding ribonucleoside 5'diphosphates (ADP, GDP, UDP, and CDP). This step is the rate-limiting reaction in the production of 2 '-deoxyribonucleoside 5'-triphosphates required for DNA replication (1). RNR consists of two protein components. R 1 is a $160-\mathrm{kDa}$ dimer that contains at least two different effector-binding sites and $\mathrm{R} 2$ is a $78-\mathrm{kDa}$ dimer that contains a non-heme iron that participates in catalysis by forming an unusual free radical on the aromatic ring of a tyrosine residue. Expression of both $\mathrm{R} 1$ and R2 are required for enzymatic activity. Interestingly, $\mathrm{R} 1$ and R2 are encoded by different genes located on separate chromosomes and the mRNAs are differentially expressed throughout the cell cycle $(2,3)$. Consequently, the level of R1 protein remains relatively stable throughout the cell cycle, while R2 is only expressed during late G1/early S phase, when DNA replication occurs. RNR activity is regulated by the amount of enzyme present in the cell and by allosteric control mechanisms involving positive and negative effectors $(1,4)$.

Recently, an R2 paralogue, p53R2, was identified that is induced by DNA damage (5-7). Expression of p53R2 is regulated by $\mathrm{p} 53$, via a 53 binding sequence in intron 1 of 
the $\mathrm{p53R} 2$ gene. UV and $\gamma$ radiation and adriamycin treatment induce p53R2 expression, but not R2, in a p53-dependent manner. In addition, p53R2 can form an active ribonucleotide reductase complex with $R 1$, suggesting that $R 1$ may be the endogenous partner of p53R2 (8). The identification of an alternate small subunit for the RNR complex may explain the previously observed differential expression of R1 and R2.

Several recent studies provide renewed interest in targeting RNR in the development of anti-cancer and antiHIV therapeutics (9-13). Antisense oligodeoxynucleotides (AS-ODN) are currently being studied for their potential use as therapeutic agents for a variety of diseases including cancer (14-16). One antisense compound, fomiversen, has been approved for use in treating cytomegalovirus-induced retinopathy in AIDS patients. AS-ODNs are short DNA molecules that can interfere with gene expression by forming duplexes with complementary sequences of target messenger RNAs (mRNAs) $(17,18)$. While the exact mechanisms involved are not understood, binding of AS-ODNs to mRNA may cause mRNA inactivation by sterically hindering mRNA interaction with ribosomes, spliceosomes and/or regulatory binding proteins. Alternatively, AS-ODN binding may induce degradation of the resulting RNA/DNA hybrid by the action of RNase H. Given the gene specific mechanism of action of such compounds it is expected that they will be less toxic than conventional chemotherapeutic agents $(15,17,18)$.

In the present study, 103 oligonucleotides complementary to R1 were screened for the ability to decrease R1 mRNA levels in vitro. One AS-ODN, GTI-2501, was further characterized in in vitro and in vivo assays for anti-tumor activity. The results presented here provide evidence that GTI-2501 acts in a sequence-specific, dose-dependent manner to downregulate $\mathrm{R} 1$ with a concomitant decrease in proliferation, tumor growth and metastasis. Given these data, GTI-2501 shows promise as an anti-tumor drug candidate.

\section{Materials and methods}

Oligonucleotide synthesis. All oligonucleotides used in this study were fully thioated. They were synthesized on an automated DNA synthesizer (Perkin-Elmer, USA) by Boston BioSystem Inc. (Boston, MA). Oligonucleotides were purified and purity was assessed by reversed-phase high performance liquid chromatography. Each oligonucleotide preparation was found to be $>95 \%$ full-length material. GTI-2501 hybridizes to the coding region of R1 mRNA. Two mismatched control analogues of GTI-2501 (GTI-2501misA and GTI-2501misB) contain 2 base changes in the middle of GTI-2501 sequences. Scrambled control analogues of GTI-2501, GTI-2501scrA-C not complementary to $\mathrm{R} 1$, while keeping the same base composition ratio as GTI-2501, were synthesized. Table I shows the sequence of each oligonucleotide and the figure legends indicate which were used for each experiment.

Cell lines and cell culture. Unless otherwise indicated in the text human tumor cell lines were purchased from the American Type Culture Collection (ATCC; Rockville, MD). Colon adenocarcinoma (HT-29), non-small cell lung carcinoma (NCIH460), melanoma (A2058), breast adenocarcinoma (MDAMB-231), pancreatic adenocarcinoma (AsPC-1, SU.86.86),
Table I. Oligonucleotide sequences.

\begin{tabular}{lc}
\hline $\begin{array}{l}\text { Oligonucleotide } \\
\text { name }\end{array}$ & \multicolumn{1}{c}{ Sequence (5'-3') } \\
\hline GTI-2501 & CTC TAG CGT CTT AAA GCC GA \\
GTI-2501misA & CTC TAG CTG CTT AAA GCC GA \\
GTI-2501misB & CTC TAG CGT CAT ATA GCC GA \\
GTI-2501scrA & ACT GCA GCC TAT ATG CAG CT \\
GTI-2501scrB & ACG CAC TTA GCT AGT GAC TC \\
GTI-2501scrC & ATG CAC TCA GCT TGT GAC AC \\
\hline
\end{tabular}

glioblastoma-astrocytoma (U-87 MG), renal carcinoma (A-498 and Caki-1), ovarian adenocarcinoma (SK-OV-3), prostate adenocarcinoma (DU 145, PC-3), hepatocellular carcinoma (Hep G2), cervical squamous carcinoma ( $\mathrm{SiHa}$ ), and Burkitt's lymphoma (Raji) cells were maintained, according to ATCC recommendation, in $\alpha$-MEM, RPMI-1640 or McCoy's 5a medium (Gibco-BRL, Gaithersburg, MD) supplemented with $10-20 \%$ fetal calf serum (FCS) at $37^{\circ} \mathrm{C}$ in a humidified atmosphere containing $5 \% \mathrm{CO}_{2}$. C8161 metastatic melanoma cells were a gift from Dr D.R. Welch, University of Pennsylvania, Hershey, PA, and were maintained as above (19). All media used in these experiments also contain an antibiotic-antimycotic solution at a final concentration of 100 units $/ \mathrm{ml}$ penicillin and $100 \mu \mathrm{g} / \mathrm{ml}$ streptomycin (Gibco-BRL). Normal human cell lines, WI-38 (human embryonic lung fibroblast) and HUVEC (human umbilical cord vascular endothelial cells) cells were maintained as above.

Oligonucleotide treatment of the cells in culture. Cells were seeded into 60 or $100 \mathrm{~mm}$ tissue culture dishes and grown to sub-confluency (75-85\%). Subsequently, the cells were washed with phosphate-buffered saline, pH 7.2 (PBS) and treated with oligonucleotides in the presence of cationic lipid (Lipofectin reagent, final concentration of $5 \mu \mathrm{g}$ of DOTMA/ $\mathrm{DOPE} / \mathrm{ml}$, Gibco-BRL) for $4 \mathrm{~h}$. After the incubation period, the media containing oligonucleotides were removed and cells were washed once with PBS. The cells were then cultured in growth medium for the duration indicated in the text.

Colony forming assay. Colony forming ability of cells treated with oligonucleotides was estimated as previously described (20). Briefly, aliquots of cell suspension were seeded into $60 \mathrm{~mm}$ tissue culture dishes at a density of $1 \times 10^{4} / \mathrm{dish}$, incubated overnight at $37^{\circ} \mathrm{C}$, washed once in $5 \mathrm{ml} \mathrm{PBS}$ and treated with oligonucleotides as above. The oligonucleotides were removed by washing once with PBS and the cells were cultured in appropriate growth medium for 7-10 days. Surviving colonies of $\geq 50$ cells were visualized by methylene blue staining and scored by direct counting $(20,21)$.

Measurement of R1 protein levels. To measure the effect of GTI-2501 on R1 protein level, Western blot analysis was conducted as previously described $(21,22)$. Briefly, cells were treated with the indicated concentrations of oligonucleotides for $4 \mathrm{~h}$, incubated for 8-18 h, washed once with PBS and whole cell protein extracts were prepared in 50-150 $\mu 1$ of 
2X sample loading buffer (100 mM Tris, pH 6.8, $200 \mathrm{mM}$ DTT, $4 \%$ SDS, $20 \%$ glycerol and $0.015 \%$ bromophenol blue). Extracted protein (10-20 $\mu \mathrm{g}$ ) was fractionated on $12 \%$ SDS-PAGE gel, transferred to nitrocellulose membranes and total protein visualized by India ink staining. R1 protein was detected with AD203, an anti-R1 antibody $(5-50 \mu \mathrm{g} / \mathrm{ml}$; obtained from either InRo BiomedTek, Sweden or Accurate Chemical and Scientific Corporation, Westbury, NY, USA) followed by horseradish peroxidase-conjugated goat antirabbit IgG (Sigma, St. Louis, MO) at a dilution of 1:5,000. The $80 \mathrm{kDa}$ R1 protein was visualized by development of the peroxidase reaction (ECL Chemiluminescence, Amersham, Arlington Heights, IL).

Immunoprecipitation was performed using saturating amount of AD203 anti-R1 monoclonal antibody as previously described (21). Briefly, cells were treated as described above, washed with PBS, labeled with ${ }^{35} \mathrm{~S}$-methionine for 4-7 $\mathrm{h}$ and cell extracts prepared by lysis in SB250 buffer $(250 \mathrm{mM}$ $\mathrm{NaCl}, 25 \mathrm{mM}$ Tris- $\mathrm{HCl}$, pH 7.5, 5 mM EDTA, 1\% Triton $\mathrm{X}-100,0.5 \%$ sodium deoxycholate). $\mathrm{R} 1$ protein was specifically immunoprecipitated by incubation with saturating amounts of R1 antibody followed by incubation with formalin-fixed staphylococcus aureas cells (Pansorbin, Calbiochem). The isolated protein was resolved on $12 \%$ SDS-PAGE gel and visualized by autoradiography.

Measurement of RI mRNA levels. To measure the effect of GTI-2501 on R1 mRNA levels, Northern blot analysis was conducted as previously described (23). Briefly, cells were treated as described above and total cellular RNA was prepared using TRIzol reagent (Gibco, Gaithersburg, MD). Total cellular RNA (10-20 $\mu \mathrm{g})$ was resolved on $1.0 \%$ denaturing formaldehyde agarose gel and transferred to nylon membrane using capillary transfer. The blots were hybridized with a ${ }^{32} \mathrm{P}$-labeled R1 fragment (24) and R1 mRNA was visualized and quantified using autoradiography or phosphorImager analysis (Molecular Dynamics, Sunnyvale, CA). Either glyceraldehyde-3-phosphate dehydrogenase (GAPDH) mRNA or ribosomal RNA levels were simultaneously probed or stained with methylene blue, respectively, for RNA loading controls.

In vivo treatment with antisense oligonucleotides. CD-1 athymic female nude mice, BALB/c nu/nu nude mice, SCID, and SCID/beige mice were purchased from Charles River Laboratories (Montreal, Canada). Unless otherwise indicated, experiments began when the mice were 6-7 weeks old. Human tumor cells were grown in appropriate growth medium and $3 \times 10^{6}-1 \times 10^{7}$ cells suspended in $100 \mu 1$ of PBS were subcutaneously injected into the right flank of the animals with a 23-gauge needle. Each experimental group typically contained 5-10 mice. Treatment started after the size of tumor reached a volume of 50-100 $\mathrm{mm}^{3}$. Antisense oligonucleotides (dissolved in saline) were administered by bolus infusion into the tail vein every other day at the indicated dose. Treatments with 5-fluorouracil (5-FU; Pharmacia), vinblastine (Faulding) and gemcitabine (Eli Lilly) were as indicated in the figure legend. Anti-tumor activity was estimated by the measurement of tumor volume with calipers at 2-day intervals. Tumor volume was calculated by a formula, $\mathrm{L} \times \mathrm{W} \times \mathrm{H} / 2$, where $\mathrm{L}$ indicates length, $\mathrm{W}$ indicates width and $\mathrm{H}$ indicates height of the tumor

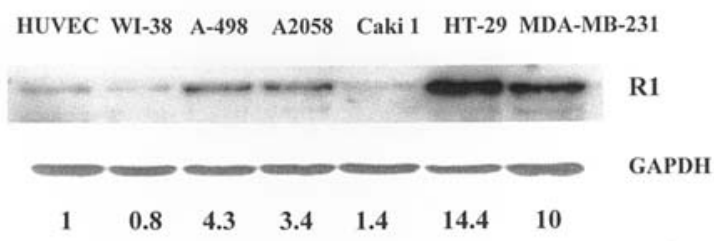

Figure 1. Over-expression of R1 protein. Two normal cell lines (HUVEC, WI-38) and 5 tumor cell lines (A-498, A2058, Caki-1, HT-29 and MDAMB-231) were analyzed by Western blotting as described in Materials and methods. The R1 protein is shown in the upper panel and GAPDH in the lower panel. The ratio of R1 to GAPDH determined by densitometry is indicated below each lane.

(25-27). Within $24 \mathrm{~h}$ after the last treatment, the animals were sacrificed and tumor and body weights were measured.

Experimental metastasis assay. C8161 human melanoma cells were seeded into $100 \mathrm{~mm}$ tissue culture dishes at a density of $2 \times 10^{6} /$ dish and incubated overnight at $37^{\circ} \mathrm{C}$ in $\alpha$-MEM medium supplemented with $10 \%$ FBS. The cells were trypsinized, collected by centrifugation and aliquots were removed from the suspension to determine cell viability using trypan blue exclusion test. Approximately $1 \times 10^{5}$ cells suspended in $0.1 \mathrm{ml}$ of PBS were injected into the tail veins of 6-8 weeks old CD-1 athymic female nude mice. Treatment, as indicated in the figure legend, was initiated after 1 day. Estimates of the number of lung nodules were made 50 days following injection of tumor cells, after excised lungs from individual mice were stained with picric acid dye solution (75\% picric acid, $20 \%$ formaldehyde, $5 \%$ glacial acetic acid).

Lymphoma survival assay. Viable human Burkitt's lymphoma (Raji) cells $\left(5 \times 10^{6}\right)$ collected from sub-confluent logarithmically growing cultures were injected intravenously into the tail vein of SCID mice and disease was allowed to establish for 2 days. Oligonucleotides, in normal saline, were administered by tail vein injections every second day at a dose of $10 \mathrm{mg} / \mathrm{kg}$. Control animals received saline alone, without oligonucleotide or scrambled control. Each treatment group typically contained 10 animals. Treatment with oligonucleotide was stopped as indicated in the figure legend. The anti-tumor efficacy of each treatment was assessed by the examination of the survival of the mice. Survival is reported as a percentage of the starting number of mice in the treatment group.

Densitometry. Results were quantified using Bio-Rad GelDoc System and Bio-Rad Quantity One quantitation software (ver. 4.3.0).

\section{Results}

R1 protein levels are elevated in cancer cell lines. Earlier studies have demonstrated elevated RNR levels and activity in tumors and tumor cell lines (28,29; Saeki et al, Proc ASCO 16, 1997). To assess whether this is a general phenomenon of cancer cells, the R1 protein levels were examined in two normal and cancer cell lines derived from diverse cancer types, including renal, skin, colon and breast cancer cell lines (Fig. 1). GAPDH protein expression was determined as an 
A

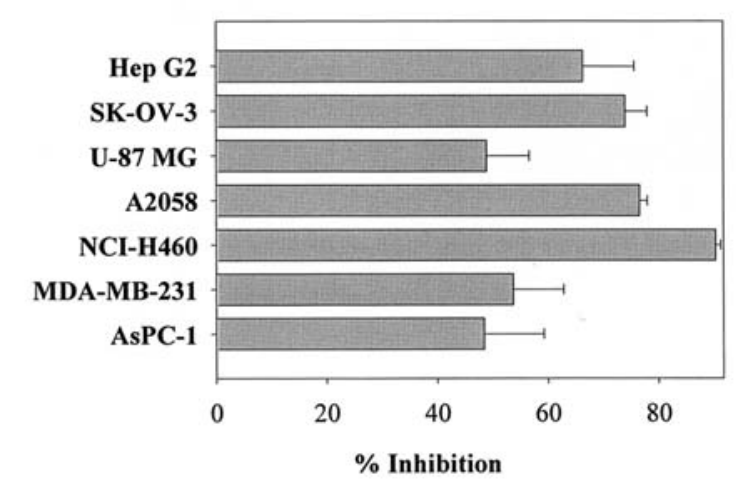

B

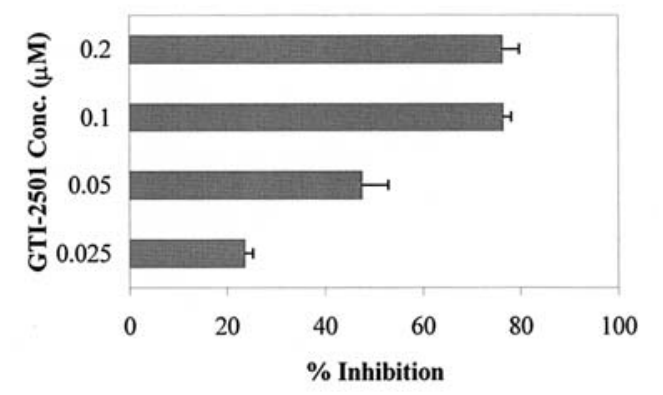

Figure 2. Inhibition of tumor cell growth by GTI-2501. (A) The effect of GTI-2501 on colony forming ability was evaluated in the following human tumor cell lines: Hep G2 (liver), SK-OV-3 (ovary), U-87 MG (brain), A2058 (melanoma), H460 (lung), MDA-MB-231 (breast) and AsPC-1 (pancreas) as described in Materials and methods. Results are summarized from 4 to 8 trials for each tumor cell line and are reported as \% inhibition compared to vehicle control. (B) The colony forming ability of MDA-MB-231 human breast adenocarcinoma cells treated with increasing concentrations of GTI2501 was assessed to determine the dose-dependent anti-proliferative property of GTI-2501. Cells were treated with $0.025,0.05,0.1$, or $0.2 \mu \mathrm{M}$ GTI-2501 as described above. Percent inhibition of colony formation was calculated by comparing the number of colonies observed in the presence of GTI-2501 with that observed in the absence of the antisense oligonucleotide. All experiments were performed in quadruplicate.

internal reference. Consistent with its role in cancer progression, R1 levels were elevated in all of the tumor cell lines tested. The increase in R1 varied from 1.4- to 14-fold, compared to HUVEC cells, and 1.8- to 17-fold, compared to WI-38 cells. In addition to the above cell lines R1 levels were assessed in Colo205, Colo320HSR, C8161, G401, HCT116, MM-RV and RD cells. R1 levels were elevated 2 - to 15 -fold in these cell lines compared to WI-38 normal cells (unpublished data). These data support the targeting of $\mathrm{R} 1$ for down-regulation via antisense compounds.

GTI-2501 displays dose-dependent anti-proliferative activity in vitro. One hundred and three oligonucleotides complementary to R1 were screened for the ability to downregulate R1 mRNA and protein expression, decrease proliferation of cancer cells in culture and decrease tumor cell growth in xenograft tumor models. In all cell lines tested one oligonucleotide, designated GTI-2501, produced at least $50 \%$ inhibition of cell proliferation as assessed by colony forming assays (Fig. 2A). Inhibition of proliferation by GTI-
A

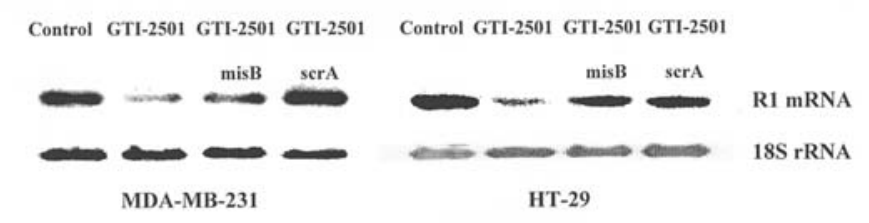

B

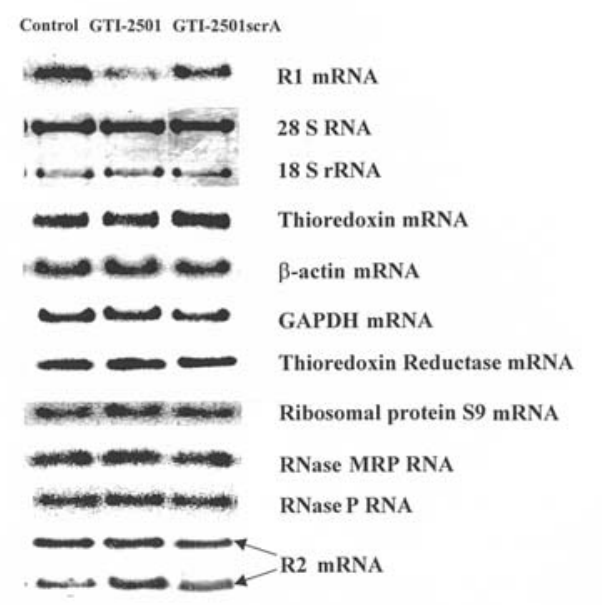

Figure 3. Sequence- and target-specific inhibition of the R1 mRNA. (A) Northern blot analysis was performed to determine if GTI-2501 treatment of human tumor cell lines had an effect on R1 mRNA levels. HT-29 and MDA-MB-231 cells were treated with Lipofectin alone or $0.2 \mu \mathrm{M}$ GTI-2501, GTI-2501misB or GTI-2501scrA (2 independent treatments) as described in Materials and methods. 18S rRNA levels were simultaneously probed for RNA loading controls (bottom panels). (B) To examine the target and sequence specificity of inhibition of R1 mRNA by GTI-2501, Northern blot analyses of other cellular RNA levels in A2058 human melanoma cells treated with GTI-2501 or a scrambled control analogue of GTI-2501 (GTI-2501scrA) were carried out. The blots were hybridized with ${ }^{32} \mathrm{P}$-labeled probes that detect R1 mRNA, 28S rRNA, 18S rRNA, thioredoxin mRNA, B-actin mRNA, GAPDH mRNA, thioredoxin reductase mRNA, ribosomal protein S9 mRNA, RNase MRP RNA, RNase P RNA and R2 mRNA.

2501 was dose-dependent reaching a maximum at $0.1 \mu \mathrm{M}$ in the breast cancer cell line MDA-MB-231 (Fig. 2B). GTI-2501 was also highly effective in down-regulating the target mRNA and protein and in decreasing growth of tumor xenografts. Although sequence specificity was not evaluated in the preliminary broad screening, the results suggested that GTI-2501 would be a good lead candidate compound for further analysis.

GTI-2501 specifically inhibits expression of R1 mRNA and protein in vivo and in vitro. A limitation of most in vitro proliferation assays is that cells in culture can be sensitive to the PS-AS-ODNs backbone (unpublished data). As such, these assays are only appropriate for excluding PS-AS-ODNs that do not demonstrate any activity prior to in vivo screening. In order to invoke an antisense mechanism of action, there must be a correlation between anti-proliferative activity and target down-regulation. HT-29 and MDA-MB-231 cells were treated with GTI-2501, mismatched and scrambled control sequences. Treatment with GTI-2501, but not control oligonucleotides resulted in a significant decrease in R1 mRNA 


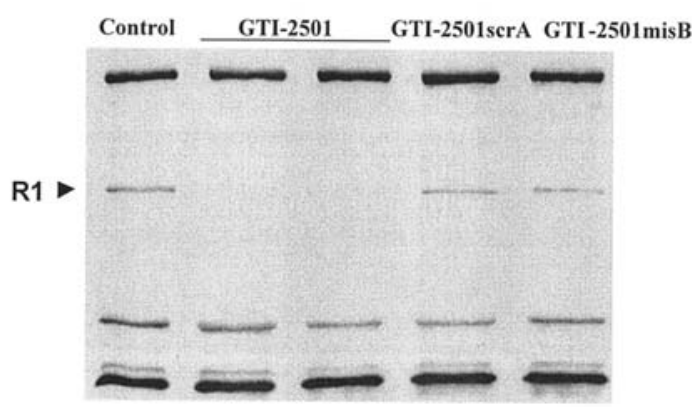

B
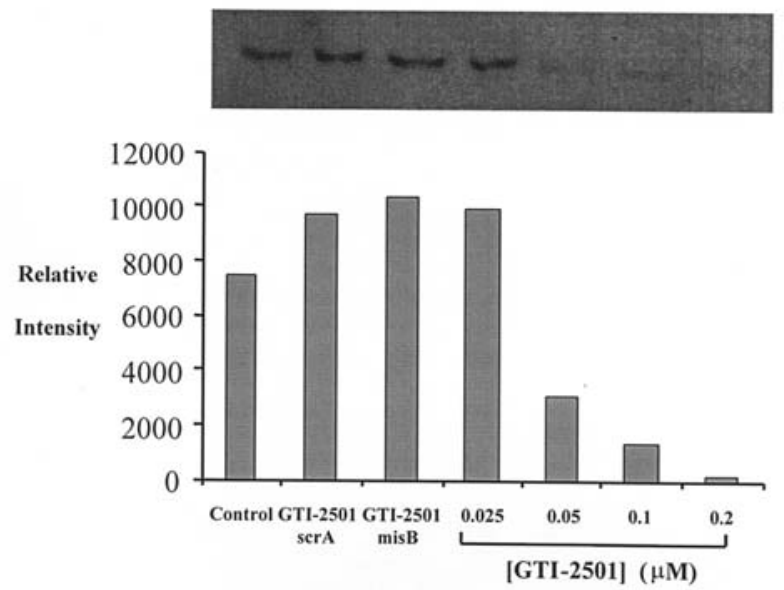

Figure 4. Sequence-specific inhibition of R1 protein biosynthesis and steady state protein levels. (A) Immunoprecipitation was performed in ${ }^{35} \mathrm{~S}$-methionine labeled AsPC-1 (pancreatic adenocarcinoma) cells treated with GTI-2501, GTI-2501misB or GTI-2501scrA as described in Materials and methods. (B) Western blot analysis was performed as described in Materials and methods, to evaluate the steady state levels of R1 from MDA-MB-231 human breast adenocarcinoma cells treated with increasing concentrations $(0.025-0.2 \mu \mathrm{M})$ of GTI-2501, $0.2 \mu \mathrm{M}$ GTI-2501scrA, $0.2 \mu \mathrm{M}$ GTI-2501misB or Lipofectin alone. The R1 (upper panel) was quantified by densitometry and the results for each treatment presented as relative intensity (bottom panel).

under conditions where internal control 18s rRNA remained constant (Fig. 3A). Fig. 3B demonstrates that GTI-2501 is both sequence and target specific. Treatment of A2058 melanoma cells with GTI-2501 resulted in a decrease in R1 mRNA while treatment with the scrambled control oligonucleotide had no effect. Furthermore, GTI-2501 specifically down-regulated R1 and not unrelated RNAs including 18S, 28S, thioredoxin mRNA, GAPDH mRNA and R2 mRNA (Fig. 3B).

To determine whether a decrease in R1 mRNA translates into decreased R1 protein synthesis, AsPC-1 cells were treated with GTI-2501 and control oligonucleotides, as above, and newly synthesized proteins were labeled with ${ }^{35} \mathrm{~S}$-methionine. R1 was isolated from cell lysates by immunoprecipitation with an R1 specific antibody. Fig. 4A clearly demonstrates that GTI-2501 down-regulates R1 protein synthesis in a sequencespecific manner as scrambled and mismatched control oligonucleotides did not down-regulate R1. Furthermore, GTI2501 , but not the control oligonucleotides, down-regulated steady state levels of $\mathrm{R} 1$ protein as assessed by Western blot analysis in MDA-MB-231 cells (Fig. 4B). GTI-2501 inhibited $\mathrm{R} 1$ expression in a dose-dependent manner that approximates the effects on proliferation (Fig. 2B).
A
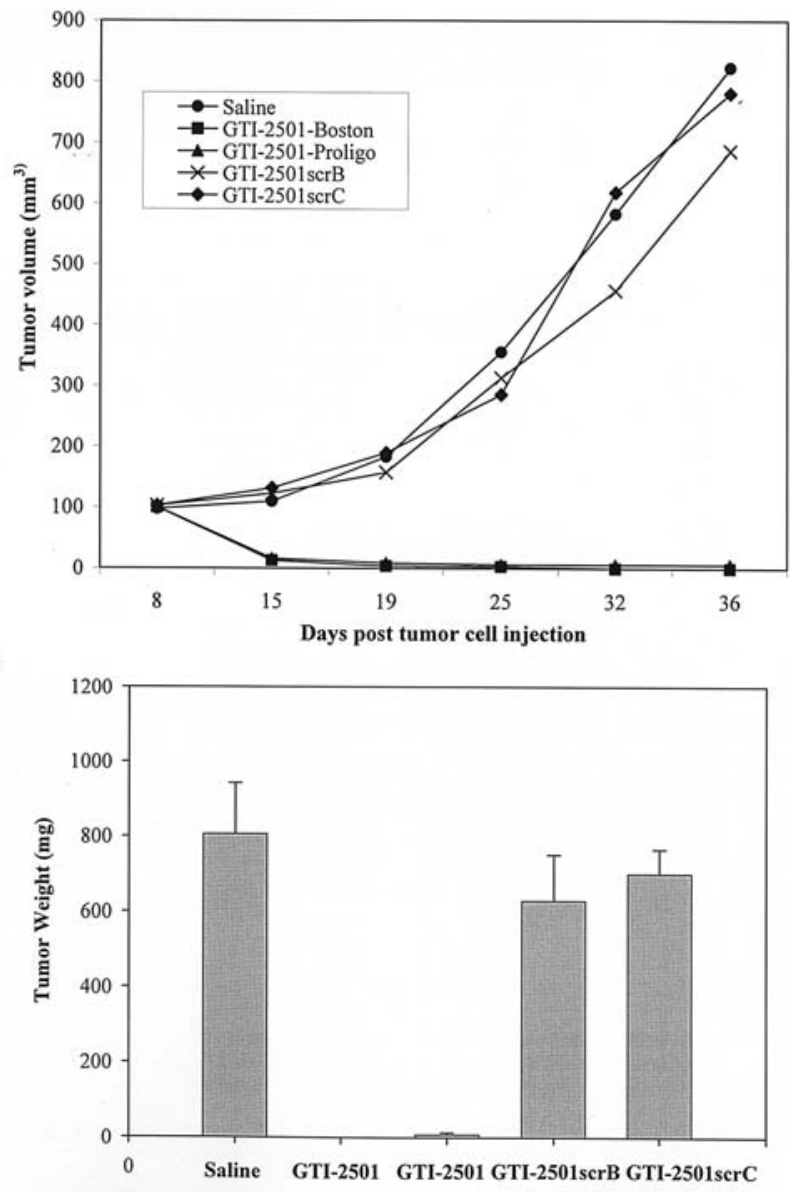

Figure 5. Sequence-specific inhibition of Caki-1 renal carcinoma tumor growth by GTI-2501. Caki-1 human renal carcinoma cells $\left(1 \times 10^{7}\right.$ cells in $100 \mu 1$ of PBS) were subcutaneously injected into the right flank of 6-7 weeks old female SCID mice. After the size of the tumors reached an approximate volume of $100 \mathrm{~mm}^{3}, 8$ days post tumor cell injection, $10 \mathrm{mg} / \mathrm{kg}$ of GTI-2501 (Proligo), GTI-2501 (Boston BioSystems), GTI-2501scrB or GTI-2501scrC were administered by bolus infusion into the tail vein every other day until the 36th day. Control animals received saline alone for the same period. (A) Anti-tumor activity was estimated by the inhibition of tumor volume, calculated from caliper measurements. Each point represents mean tumor volume calculated from 5 animals per experimental group. P-values compared to saline: GTI-2501 (Boston) $\mathrm{P}<0.0001$, GTI-2501 (Proligo) $\mathrm{P}<0.0001$, GTI$2501 \mathrm{scrB} P=0.0858$, GTI-2501scrC $\mathrm{P}=0.7279$. (B) At the end of treatment, the animals were sacrificed, tumors were excised and their weights were measured. Each bar represents mean tumor weight calculated from 5 animals per experimental group. P-values compared to saline: GTI-2501 (Boston) $\mathrm{P}<0.0001$, GTI-2501 (Proligo) $\mathrm{P}<0.0001$, GTI-2501scrB P=0.3591, GTI2501 scrC $\mathrm{P}=0.5011$.

The observed sequence-specific down-regulation of R1 in a variety of cancer cell lines suggested that GTI-2501 could have broad anti-cancer activity in vivo.

GTI-2501 displays sequence-specific and dose-dependent anti-tumor activity against human tumor cell xenografts. The sequence-specific anti-tumor activity of GTI-2501 is demonstrated in Fig. 5. The anti-tumor activity of two preparations of GTI-2501 (manufactured by Proligo and Boston BioSystems) was compared to control treatments (saline and two scrambled controls). Growth of renal tumor xenografts was dramatically reduced in both GTI-2501 treatment groups, with complete regression in all but 2 of 10 mice. In contrast, tumor growth in the GTI-2501scr treatment 
A

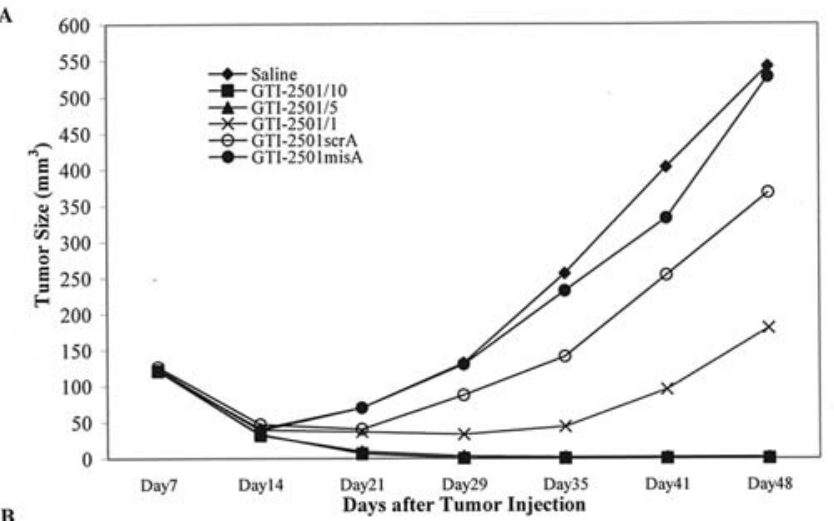

B

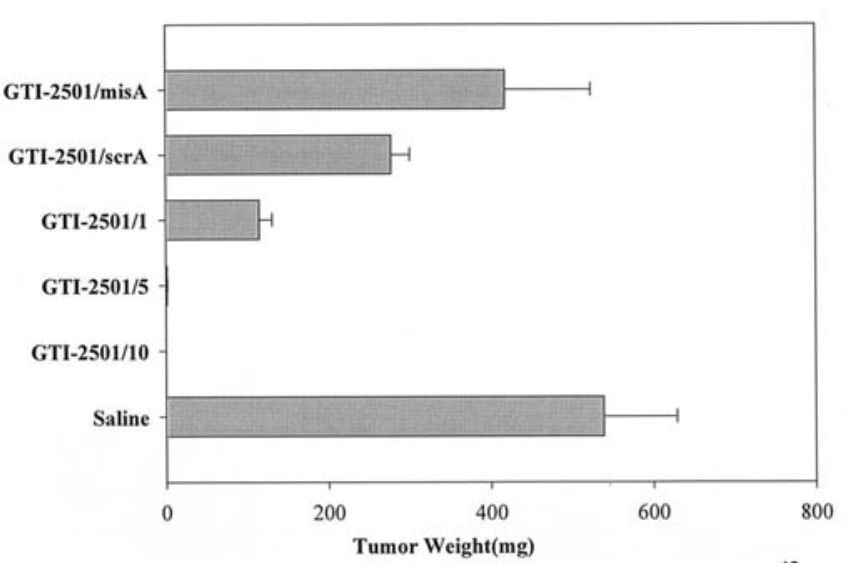

Figure 6. Dose-dependent effects of GTI-2501 on Caki-1 renal carcinoma growth in SCID mice. Caki-1 human renal carcinoma cells $\left(1 \times 10^{7}\right.$ cells in $100 \mu 1$ of PBS) were subcutaneously injected into the right flank of 6-7 weeks old female SCID mice. After the size of the tumors reached an approximate volume of $100 \mathrm{~mm}^{3}, 7$ days post tumor cell injection, increasing concentrations (1.0-10 mg/kg, designated as GTI2501/1 to 10) of GTI-2501 were administered by bolus infusion into the tail vein every other day for 40 days. GTI-2501misA and GTI-2501scrA were administered at a dose of $10 \mathrm{mg} / \mathrm{kg}$ every other day. Control animals received saline alone for the same period. (A) Anti-tumor activity was estimated by the inhibition of tumor volume, calculated from caliper measurements. Each point represents mean tumor volume calculated from 10 animals per experimental group. P-values compared to saline: GTI- $2501,1,5,10 \mathrm{mg} / \mathrm{kg}$ P $<0.0001$, GTI$2501 \mathrm{misA} P=0.5854$, GTI-2501scrA $P=0.2103$. (B) At the end of treatment, the animals were sacrificed, tumors were excised and their weights were measured. Each bar represents mean tumor weight calculated from 10 animals per experimental group. P-values compared to saline: GTI-2501, 1, 5, $10 \mathrm{mg} /$ kg P<0.0001, GTI-2501misA $\mathrm{P}=0.3884$, GTI-2501scrA $\mathrm{P}=0.0791$.

groups was indistinguishable from that in the saline control group.

To determine whether GTI-2501 acts in a dose-dependent manner in vivo, mice bearing Caki-1 renal xenografts were treated with increasing amounts of GTI-2501 and tumor growth was assessed by measurement of tumor volume and weight. Doses of as little as $1 \mathrm{mg} / \mathrm{kg}$ every other day were sufficient to reduce tumor growth significantly (Fig. 6). At a dose of $5 \mathrm{mg} / \mathrm{kg}$ every other day the tumors were stabilized and some regressed while at the maximum dose of $10 \mathrm{mg} / \mathrm{kg}$ every other day there was complete tumor regression in all treated mice by day 29. Scrambled and mismatched control oligonucleotides, at a dose of $10 \mathrm{mg} / \mathrm{kg}$, did not have significant anti-tumor activity. Taken together these results demonstrate that GTI-2501 has sequence-specific anti-tumor activity.
A

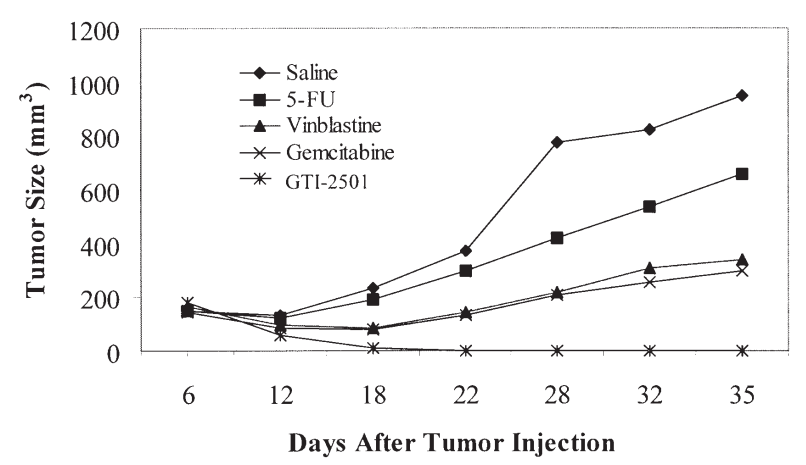

B

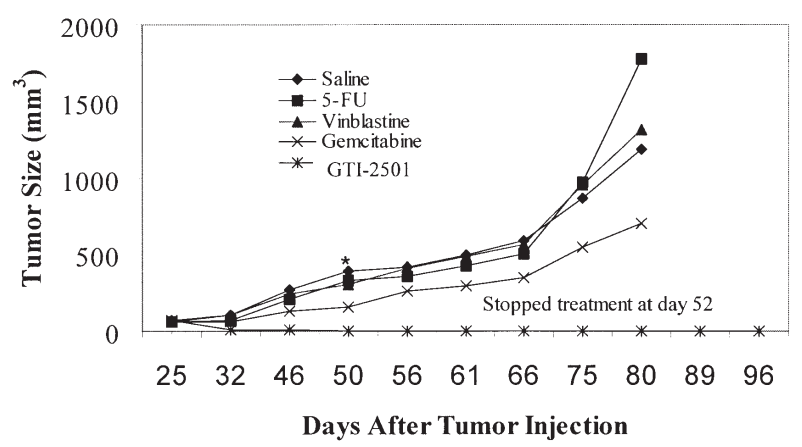

Figure 7. Comparison of GTI-2501 to 5-FU, vinblastine and gemcitabine in inhibition of renal tumor growth. (A) Caki-1 human kidney cancer cells ( $1 \times 10^{7}$ cells in $100 \mu 1$ of PBS) were subcutaneously injected into the right flank of 6-7 weeks old female SCID mice. After the size of the tumors reached an approximate volume of $150 \mathrm{~mm}^{3}, 6$ days post tumor cell injection, GTI2501 was administered by bolus infusion into the tail vein every other day at $10 \mathrm{mg} / \mathrm{kg}$. Control animals received saline alone for the same period. The anti-tumor effect of GTI-2501 was further compared to that of three chemotherapeutic agents including 5-FU, vinblastine and gemcitabine. 5-FU was administered intraperitoneally at days 7-13, 21-27 and 35-36 with a dose of $13 \mathrm{mg} / \mathrm{kg} /$ day, while vinblastine and gemcitabine were administered intraperitoneally at days $7,14,21,28$ and 35 at a dose of $0.6 \mathrm{mg} / \mathrm{kg} /$ week or $80 \mathrm{mg} / \mathrm{kg} /$ week, respectively. All treatments were stopped at day 36. Antitumor activities were estimated by the inhibition of tumor volume, calculated from caliper measurements. Each point represents mean tumor volume calculated from 5 animals per experimental group. P-values compared to saline: GTI-2501 $\mathrm{P}=0.0001$, gemcitabine $\mathrm{P}=0.0001$, vinblastine $\mathrm{P}=0.0001$, 5 -FU $\mathrm{P}=0.0002$. (B) A-498 human kidney cancer cells $\left(1 \times 10^{7}\right.$ cells in $100 \mu 1$ of PBS) were subcutaneously injected into the right flank of 6-7 weeks old female SCID mice. After the size of the tumors reached an approximate volume of $100 \mathrm{~mm}^{3}, 25$ days post tumor cell injection, GTI-2501 was administered by bolus infusion into the tail vein every other day at $10 \mathrm{mg} / \mathrm{kg}$. Control animals received saline alone for the same period. The anti-tumor effect of GTI-2501 was further compared to that of three chemotherapeutic agents including 5-FU, vinblastine and gemcitabine. 5-FU was administered intraperitoneally at days 26-32, and 39-46 with a dose of $13 \mathrm{mg} / \mathrm{kg} / \mathrm{day}$, while vinblastine and gemcitabine were administered intraperitoneally at days $26,32,39,46$ and 52 at a dose of $0.6 \mathrm{mg} / \mathrm{kg} /$ week or $80 \mathrm{mg} / \mathrm{kg} /$ week, respectively. All treatments were stopped at day 52 . Mice treated with saline or the three chemotherapeutic agents were sacrificed at day 80 because the tumor sizes became too large. Mice treated with GTI-2501 were kept for 44 more days to observe possible resurgence of resistant tumor growth. Antitumor activities were estimated by the inhibition of tumor volume, calculated from caliper measurements. Each point represents mean tumor volume calculated from 5 animals per experimental group. P-values compared to saline: GTI-2501 $\mathrm{P}=0.0001$, gemcitabine $\mathrm{P}=0.0001$, vinblastine $\mathrm{P}=0.4714$, 5-FU $\mathrm{P}=0.0442$. $\mathrm{P}$-values of GTI-2501 compared to vinblastine, gemcitabine or 5 -FU were $\mathrm{P}=0.0001$.

Although it would be ideal to demonstrate target downregulation in these experiments, tumor shrinkage in the GTI- 


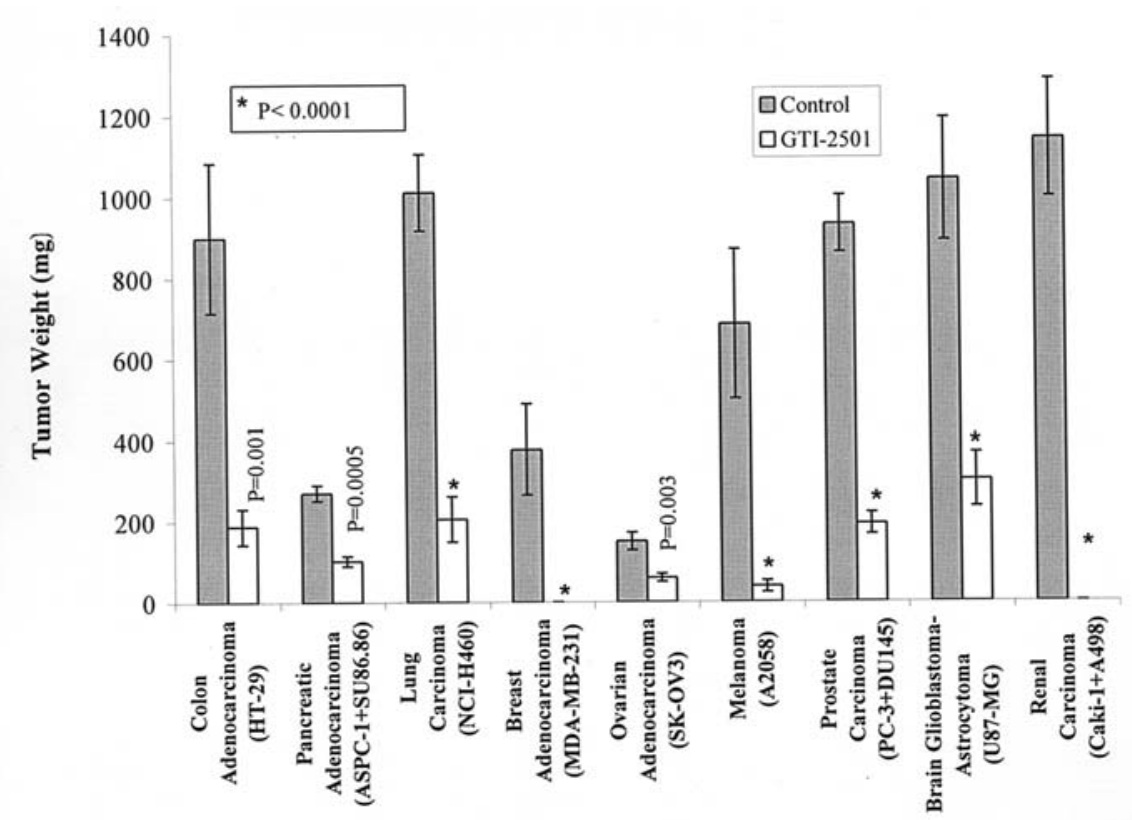

Figure 8. Summary of tumor growth assays. Tumor cells $\left(3 \times 10^{6}-1 \times 10^{7}\right.$ cells in $100 \mu 1$ of PBS) were subcutaneously injected into the right flank of mice. After the size of the tumors reached an approximate volume of $50-100 \mathrm{~mm}^{3}$, GTI-2501 was administered by bolus infusion into the tail vein at concentrations ranging from 1.0 to $10 \mathrm{mg} / \mathrm{kg}$. Control animals received saline alone for the same period. Anti-tumor activities were estimated by the inhibition of tumor weight, which was measured at the end of each treatment after the animals were sacrificed and tumors were excised. Each bar represents mean tumor weight calculated from culmination of a number of independent experiments for each tumor type. For most tumor types, a single tumor cell line was used to induce tumor growth, except pancreatic adenocarcinoma, prostate carcinoma and renal carcinoma where two independent cell lines were used.

2501-treated mice prevented the collection of sufficient tumor tissue for analysis. Furthermore, the target site in R1 is not conserved between mice and humans thereby precluding the use of mouse liver or spleen as surrogate tissue. Northern blot analysis of RNA extracted from HT-29 tumor xenografts demonstrated decreased R1 mRNA levels in GTI-2501-treated mice compared to saline-treated controls (unpublished data).

GTI-2501 is more effective than standard chemotherapeutic agents against renal tumor xenografts. The anti-tumor efficacy of GTI-2501 was compared to those of 5-FU, gemcitabine and vinblastine for two reasons. First, treatment with current therapeutic agents would assess whether the effects of GTI-2501 on renal tumor growth is simply a reflection of these tumors having increased sensitivity to cytostatic and cytotoxic agents. Second, direct comparison to other drugs in clinical use would provide an indication of whether GTI-2501 has efficacy that warrants further drug development. GTI-2501 was the only treatment that resulted in complete regression of renal tumor xenografts (Fig. 7). Furthermore, GTI-2501 anti-tumor efficacy was significantly better than that of either 5-FU, gemcitabine and vinblastine. Treatment with 5-FU, vinblastine and gemcitabine appear to become more effective over time, consistent with clinical experience with these drugs. In order to assess whether GTI-2501-induced tumor regression is long-lived, treatment of mice bearing A-498 xenografts was stopped at day 52 and mice were monitored for an additional 44 days. There were no tumor re-growths suggesting that GTI-2501 treatment cleared all tumor cells.

GTI-2501 displays anti-tumor activity against a wide range of solid tumor xenografts in vivo. Fig. 8 summarizes the results of a number of experiments that assessed the anti-tumor efficacy of GTI-2501 against 12 different tumor cell xenografts falling into 9 categories of tumor types. GTI-2501-treated mice had significantly lower tumor weights as compared to saline-treated controls. The anti-tumor efficacy ranged from approximately a 2-fold reduction in tumor size for ovarian tumors to complete regression of renal and breast tumors.

GTI-2501 treatment prolongs survival in a xenograft model of leukemia and lymphoma. In addition to anti-tumor efficacy against solid tumors, GTI-2501 was tested in a human lymphoma experimental model. Mice injected with human Burkitt's lymphoma (Raji) cells rapidly developed disease and died within 28 days of injection when treated with saline or scrambled control oligonucleotide (Fig. 9A). In contrast, treatment with GTI-2501 resulted in a dramatic increase in the survival of mice. Long-term survival of mice treated with GTI-2501 did not require ongoing treatment since discontinuation of GTI-2501 treatment at day 42 did not result in re-establishment of the disease for up to 36 days after treatment ended. In fact, GTI-2501-treated mice surviving to the end of the treatment period were sacrificed due to animal housing limitations and not disease progression. GTI-2501 treatment also dramatically prolonged the survival of mice injected with murine erythroleukemia (CB7) cells (data not shown). Saline-treated mice died between days 25 and 35 while $80 \%$ of GTI-2501-treated mice survived to the end of the 75 day study, at which time they were sacrificed due to animal housing limitations and not disease progression (data not shown).

GTI-2501 treatment decreases lung nodule formation in an experimental model of metastasis. Finally, experiments 


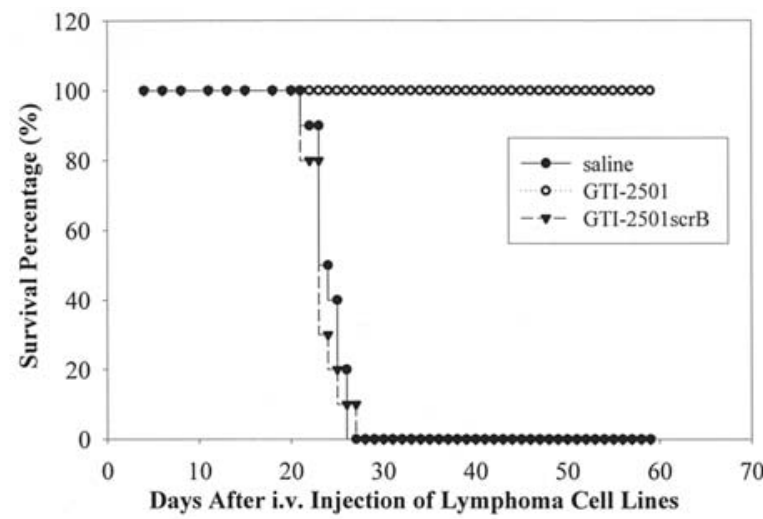

B

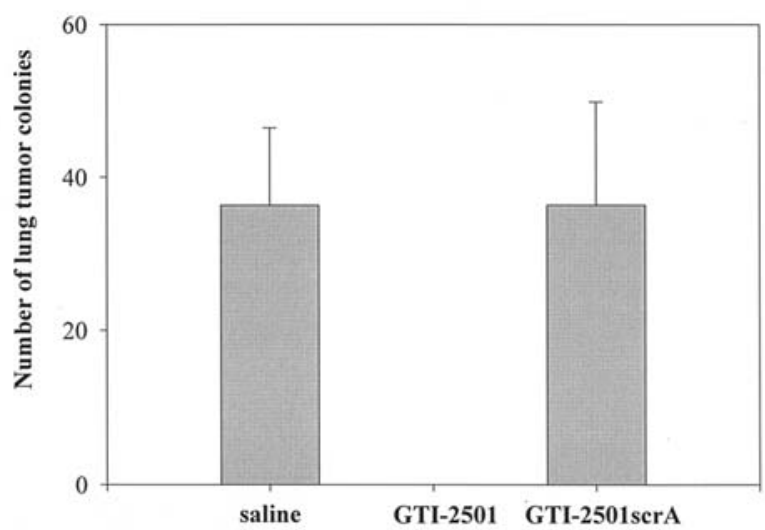

Figure 9. Anti-cancer efficacy in experimental models of lymphoma and metastasis. (A) The lymphoma survival assay was conducted as described in Materials and methods with GTI-2501, GTI-2501scrB or saline without oligonucleotide. The saline and GTI-2501scrB-treated mice died from disease progression by day 28 . Treatment with GTI-2501 was stopped at day 42 . All GTI-2501-treated mice survived to the end of the experimental period at day 78 (data not shown). (B) The lymphoma survival assay was conducted as described in Materials and methods with saline or $10 \mathrm{mg} / \mathrm{kg} / 48 \mathrm{~h}$ GTI2501 or GTI-2501scrA. The bars represent the mean number of lung nodules per mouse \pm standard error. In the GTI-2501 treatment group there were no visible lung nodules.

assessed the effects of GTI-2501 on development of tumor nodules on the lungs of mice injected with highly metastatic human melanoma (C8161) cells. Initially cultured cells were treated ex vivo for $4 \mathrm{~h}$, with GTI-2501 or Lipofectin vehicle alone, prior to injection into the tail vein of mice. After 4 weeks the mice were sacrificed, lungs excised and nodules on the lung surface counted. GTI-2501 but not the vehicle significantly reduced formation of lung nodules (unpublished data). To assess the ability of GTI-2501 to reduce metastasis in an in vivo model that more accurately reflects clinical practice, C8161 cells were first injected via the tail vein and mice were subsequently treated with saline, GTI-2501scrA or GTI-2501 after one day (Fig. 9B). In vivo treatment with GTI-2501 was as efficacious as ex vivo treatment, virtually eliminating nodule formation.

GTI-2501 does not appear to function via CpG-mediated immune stimulation. GTI-2501 contains a CpG motif that is predicted to be immuno-stimulatory (30). Given the dramatic anti-tumor effects seen in SCID (T and B cell deficient) mice, a significant CpG-mediated effect is not expected. In

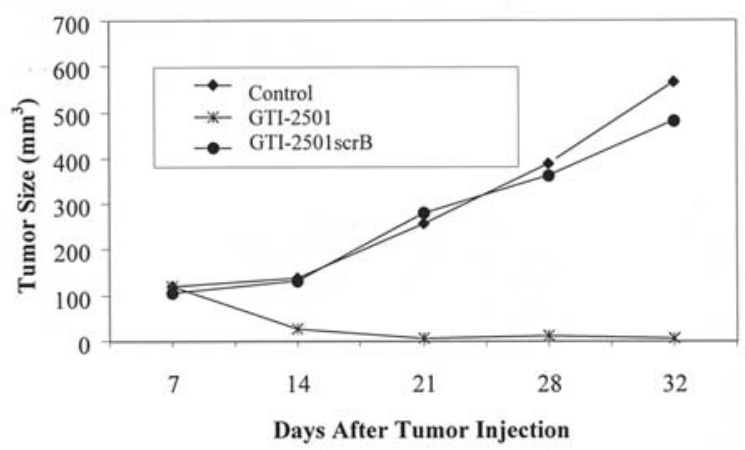

B

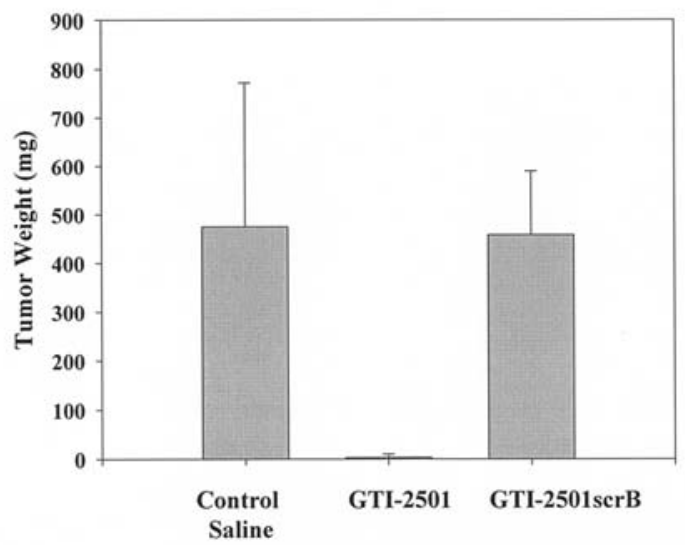

Figure 10. Inhibition of Caki-1 renal carcinoma tumor growth by GTI-2501 in SCID/beige mice. The experiments with Caki-1 renal xenografts in SCID/beige mice were essentially the same as described for SCID mice (Figs. 5-7). Each treatment group was composed of 10 mice and the data points reflect the mean of each treatment group. (A) Caliper measurements at 1-week intervals were used to calculate tumor volumes. P-values compared to saline: GTI-2501 P=0.0001, GTI-2501scrB P=0.9621. P-value of GTI-2501 compared to GTI-2501scrB was $\mathrm{P}=0.0001$. (B) After 32 days the mice were sacrificed and the tumors weighed. Each bar represents the mean tumor weight and standard error calculated for each treatment group. P-values compared to saline: GTI-2501 P=0.0001, GTI-2501scrB P=0.8357. P-value of GTI-2501 compared to GTI-2501scrB was $\mathrm{P}=0.0001$.

addition, CpG-mediated effects are not generally observed under conditions of systemic administration with saline as the vehicle. To eliminate the possibility that GTI-2501 activates NK mediated anti-tumor activity, xenograft tumor growth experiments were repeated in SCID/beige mice (Fig. 10). Treatment with GTI-2501 resulted in regression of human Caki-1 renal xenografts in SCID/beige mice in spite of the lack of NK, B or T cell function (Fig. 10). Taken together these results suggest that the observed anti-tumor activity of GTI-2501 is not attributable to CpG-mediated immune stimulation.

\section{Discussion}

With the rapid expansion in understanding the underlying mechanisms involved in initiation and progression of cancer comes the opportunity to tailor drug development to the specific pathways involved in tumorigenesis thereby decreasing toxicity associated with current chemotherapeutics. Antisense compounds as a class, are uniquely suited to this emerging drug development trend. Antisense compounds 
directly target mRNA and direct their degradation and inactivation. An oligonucleotide consisting of 20 nucleotides is sufficient to down-regulate target mRNA and at the same time is of sufficient length to provide exquisite specificity for the intended target. Furthermore, the availability of genome sequence allows for the selection of oligonucleotides that are unique to the intended target. Although clinical toxicities have been reported for antisense compounds, they are mild, reversible and associated with levels that are well above the therapeutically required dose. In addition, the administration schedule can be adjusted such that dose-limiting toxicities are minimized. Finally, oligonucleotide synthesis is scalable and becoming a financially feasible therapeutic option.

In choosing an appropriate target for down-regulation several factors should be considered. Expression of the target should be directly involved in tumor cell survival, growth or metastasis. Although not strictly required, there should be evidence for altered expression compared to normal tissues or at least there should be evidence for cancer cells having preferential requirements for the target such that a therapeutic window is available within which normal cell function is not significantly impaired. RNR, as a target fits the above criteria. Conversion of NTPs to dNTPs absolutely requires RNR and as such RNR sits as a gatekeeper to DNA synthesis and cell cycle progression. While normal cells require RNR activity there are few cells that are actively proliferating in adult tissues at a rate that approaches that of transformed cells. As a result cancer cells are likely to be differentially sensitive to loss of RNR activity. This is demonstrated by the activity of drugs such as hydroxyurea, gemcitabine and fluradabine that target RNR as part of their mechanism of action. Toxicity associated with these drugs is a result of low specificity for the therapeutic target resulting in pleiotropic effects in normal cells. The ability to specifically target the RNR complex would be expected to decrease toxicity while maintaining efficacy. Furthermore, long-term treatment with antisense compounds would not be expected to result in drug resistance as is commonly found with other RNR based therapeutics.

From a screen of PS-AS-ODNs encompassing the whole of the R1 mRNA sequence, GTI-2501 was chosen as a lead compound. The results of in vitro and in vivo experiments with GTI-2501 demonstrate sequence and target specific downregulation of R1 mRNA and protein expression. In addition to target down-regulation GTI-2501 effectively blocked growth of human tumor xenografts in mice. Anti-tumor efficacy was not observed with control oligonucleotides, indicating that GTI2501 acts via a sequence dependent mechanism of action. Taken together the in vitro and in vivo data are consistent with GTI-2501 acting via an antisense mechanism of action.

GTI-2501 has a CpG containing hexameric sequence that matches the motif expected to be immune stimulatory in mice (30). The in vivo experiments presented here were in CD-1 nude and SCID mice, which are immune deficient ( $\mathrm{T}$ cell and $\mathrm{B} / \mathrm{T}$ cell deficient respectively), suggesting that acquired immune responses are not required for GTI-2501 anti-tumor activity. To address whether there is NK mediated anti-tumor activity, the xenograft growth assay was repeated in SCID/ beige mice lacking NK, B and T cells. GTI-2501 demonstrated anti-tumor efficacy that was similar in kinetics and scale to what is observed in SCID mice suggesting innate immunity through NK cells is also not a significant factor in GTI-2501 anti-tumor efficacy (Figs. 6, 7 and 10). While there remains the possibility of an immune stimulatory component to GTI-2501 anti-tumor efficacy in immune competent subjects, the evidence would suggest that it would be secondary to sequence-specific effects.

Antisense compounds are potentially superior to conventional drugs for a number of reasons even when acting on the same target. Unlike current RNR-based drugs, GTI-2501 has the advantage of being highly specific and as a result is very well tolerated at therapeutically relevant dosages. A direct comparison of GTI-2501 to vinblastine, gemcitabine and 5-FU also demonstrates that this compound has superior anti-tumor efficacy in a human renal tumor xenograft model. GTI-2501 is the only compound in these studies that caused tumor regression of two renal tumor xenografts (A-498 and Caki-1). GTI-2501 treatment resulted in long-term protection from tumor re-growth even after treatment was stopped. In contrast, vinblastine, gemcitabine and 5-FU had decreased efficacy with no evidence of tumor stabilization or regression at therapeutically relevant dosages.

GTI-2501 efficacy was not limited to a subset of tumor types or even to a single experimental model of cancer. GTI2501 demonstrated efficacy against a wide range of solid tumors including breast, ovarian, prostate, colon, pancreatic, lung, skin and brain. In addition, GTI-2501 was effective against leukemia and lymphoma, with a dramatic increase in the survival of mice compared to control treatments. Finally, treatment with GTI-2501, ex vivo and in vivo, but not the scrambled control oligonucleotides, virtually eliminated the formation of tumor nodules on the lungs of mice following injection of highly metastatic melanoma cells into mice via the tail vein. The ability of GTI-2501 treatment to effectively target cancer in a number of different experimental models is likely a reflection of the ubiquitous nature of the target. Given that RNR is not tumor-type specific one would expect GTI-2501 to be effective in a number of cancer models. Although R1 is up-regulated in a number of cancer cell lines, over-expression is not a requirement for it being a good anticancer target. This is in contrast to other antisense compounds currently in development that targets only a subset of tumor types in which the target gene is up-regulated (15).

Although R1 levels were elevated in a number of cell lines there was no correlation between R1 levels and GTI-2501 efficacy. GTI-2501 given at $10 \mathrm{mg} / \mathrm{kg} / 48 \mathrm{~h}$ results in almost complete down-regulation of R1 mRNA in HT-29 cells, which express the highest levels of $\mathrm{R} 1$, suggesting that the therapeutic dose is sufficient for all tumors tested. Alternatively, there may be differences in how well GTI-2501 is transported into target cells and as such it would be difficult to predict tumor sensitivity based on target mRNA levels. A study by Miraglia et al, demonstrated that the target mRNA level can vary greatly with little difference in the efficacy of antisense down-regulation (31). This may be a reflection of an excess of ODN present in the cells (31). In addition, RNAse Hmediated cleavage may adjust to increasing RNA/DNA duplex formation such that differences in mRNA target levels do not limit degradation.

In addition to stalling proliferation via inhibition of RNR, down-regulation of R1 expression may also impact DNA 
repair via inhibition of p53R2-mediated dNTP supply to the repair machinery (5-7). p53R2 can form an active ribonucleotide reductase complex with $\mathrm{R} 1$ to generate an acute supply of dNTPs required for DNA repair (8). RNR R2 is not up-regulated during DNA damage and as a result supply of dNTPs is mediated via p53-regulated p53R2. The requirement of RNR R1 for both DNA replication and repair is consistent with its expression across the entire cell cycle, unlike RNR $\mathrm{R} 2$, which is tightly regulated. Radiation treatment and many chemotherapeutic agents lead to DNA damage. Consequently, R1 down-regulation may sensitize cells to apoptosis-inducing DNA damaging agents by limiting dNTP pools required for DNA repair. Studies are underway to assess the role of R1 in DNA repair and chemo-sensitization.

GLP toxicology and pharmacokinetic studies in rats and monkeys demonstrate that GTI-2501 is similar to other PSAS-ODNs and as a result support the safety of GTI-2501 (unpublished data). As a result of promising pre-clinical studies and GLP toxicology studies a phase I trial was initiated in patients with solid tumor malignancies or lymphoma for which there is no current treatment or for whom all available treatments have failed. In conclusion, the results presented in this study strongly support the development of GTI-2501 as a target-specific agent against a broad range of cancers.

\section{Acknowledgments}

We would like to thank the staff at Lorus Therapeutics Inc. for critical reading of the manuscript.

\section{References}

1. Cory JG: Purine and pyrimidine nucleotide metabolism. In: Biochemistry with Clinical Correlations. Devlin T (ed). WileyLiss, New York, pp489-523, 1997.

2. Engstrom Y, Eriksson S, Jildevik I, Skog S, Thelander L and Tribukait B: Cell cycle-dependent expression of mammalian ribonucleotide reductase. J Biol Chem 260: 9114-9116, 1985

3. Tonin PN, Stallings RL, Carman MD, Bertino JR, Wright JA, Srinivasan PR and Lewis WH: Chromosomal assignment of amplified genes in hydroxyurea-resistant hamster cells. Cytogenet Cell Genet 45: 102-108, 1987.

4. Nocentini G: Ribonucleotide reductase inhibitors: new strategies for cancer chemotherapy. Crit Rev Oncol Hematol 22: 89-126, 1996.

5. Nakano K, Balint E, Ashcroft $\mathrm{M}$ and Vousden KH: A ribonucleotide reductase gene is a transcriptional target of p53 and p73. Oncogene 19: 4283-4289, 2000.

6. Tanaka H, Arakawa H, Yamaguchi T, Shiraishi K, Fukuda S, Matsui K, Takei Y and Nakamura Y: A ribonucleotide reductase gene involved in a p53-dependent cell-cycle checkpoint for DNA damage. Nature 404: 42-49, 2000

7. Yamaguchi T, Matsuda K, Sagiya Y, Iwadate M, Fujino MA, Nakamura Y and Arakawa H: p53R2-dependent pathway for DNA synthesis in a p53-regulated cell cycle checkpoint. Cancer Res 61: 8256-8262, 2001.

8. Guittet O, Hakansson P, Voevodskaya N, Fridd S, Graslund A, Arakawa H, Nakamura Y and Thelander L: Mammalian p53R2 protein forms an active ribonucleotide reductase in vitro with the R1 protein, which is expressed both in resting cells in response to DNA damage and in proliferating cells. J Biol Chem 276: 40647-40651, 2001.

9. Crenshaw TR and Cory JG: Overexpression of protein disulfide isomerase-like protein in a mouse leukemia L1210 cell line selected for resistance to 4-methyl-5-amino-1-formylisoquinoline thiosemicarbazone, a ribonucleotide reductase inhibitor. Adv Enzyme Regul 42: 143-157, 2002.

10. Fagny C, Vandevelde M, Svoboda M and Robberecht P: Ribonucleotide reductase and thymidine phosphorylation: two potential targets of azodicarbonamide. Biochem Pharmacol 64: 451-456, 2002 .
11. Feun L, Modiano M, Lee K, Mao J, Marini A, Savaraj N, Plezia P, Almassian B, Colacino E, Fischer J and MacDonald S: Phase I and pharmacokinetic study of 3-aminopyridine-2-carboxaldehyde thiosemicarbazone (3-AP) using a single intravenous dose schedule. Cancer Chemother Pharmacol 50: 223-229, 2002.

12. Gao Y, Liehr S and Cooperman BS: Affinity-driven selection of tripeptide inhibitors of ribonucleotide reductase. Bioorg Med Chem Lett 12: 513-515, 2002.

13. Green DA, Antholine WE, Wong SJ, Richardson DR and Chitambar CR: Inhibition of malignant cell growth by 311 , a novel iron chelator of the pyridoxal isonicotinoyl hydrazone class: effect on the R2 subunit of ribonucleotide reductase. Clin Cancer Res 7: 3574-3579, 2001.

14. Braasch DA and Corey DR: Novel antisense and peptide nucleic acid strategies for controlling gene expression. Biochemistry 41: 4504-4510, 2002.

15. Flaherty KT, Stevenson JP and O'Dwyer PJ: Antisense therapeutics: lessons from early clinical trials. Curr Opin Oncol 13: 499-505, 2001

16. Opalinska JB and Gewirtz AM: Nucleic-acid therapeutics: basic principles and recent applications. Nat Rev Drug Discov 1: 503-514, 2002.

17. Ho PTC and Parkinson DR: Antisense oligonucleotides as therapeutics for malignant disease. Semin Oncol 24: 187-202, 1997.

18. Alama A, Barbieri F, Cagnoli M and Schettini G: Antisense oligonucleotides as therapeutic agents. Pharmacol Res 36: 171-178, 1997.

19. Welch DR, Bisi JE, Miller BE, Conaway D, Seftor EA, Yohem KH, Gilmore LB, Seftor RE, Nakajima M and Hendrix MJ: Characterization of a highly invasive and spontaneously metastatic human malignant melanoma cell line. Int $\mathbf{J}$ Cancer 47: 227-237, 1991.

20. Huang A and Wright JA: Fibroblast growth factor mediated alterations in drug resistance, and evidence of gene amplification. Oncogene 9: 491-499, 1994.

21. Choy BK, McClarty GA, Chan AK, Thelander L and Wright JA: Molecular mechanisms of drug resistance involving ribonucleotide reductase: hydroxyurea resistance in a series of clonally related mouse cell lines selected in the presence of increasing drug concentrations. Cancer Res 48: 2029-2035, 1988.

22. Fan H, Villegas C and Wright JA: Ribonucleotide reductase R2 component is a novel malignancy determinant that cooperates with activated oncogenes to determine transformation and malignant potential. Proc Natl Acad Sci USA 93: 14036-14040, 1996.

23. Hurta RA and Wright JA: Malignant transformation by H-ras results in aberrant regulation of ribonucleotide reductase gene expression by transforming growth factor-beta 1. J Cell Biochem 57: 543-556, 1995.

24. McClarty GA, Chan AK, Engstrom Y, Wright JA and Thelander L: Elevated expression of M1 and M2 components and drug-induced post-translational modulation of ribonucleotide reductase in a hydroxyurea-resistant mouse cell line. Biochemistry 26: 8004-8011, 1987

25. Fan H, Villegas C, Huang A and Wright JA: The mammalian ribonucleotide reductase $\mathrm{R} 2$ component cooperates with a variety of oncogenes in mechanisms of cellular transformation. Cancer Res 58: 1650-1653, 1998 .

26. Fan H, Villegas C, Huang A and Wright JA: Suppression of malignancy by the 3' untranslated regions of ribonucleotide reductase R1 and R2 messenger RNAs. Cancer Res 56: 4366-4369, 1996.

27. Damen JE, Tagger AY, Greenberg AH and Wright JA: Generation of metastatic variants in populations of mutator and amplificator mutants. J Natl Cancer Inst 81: 628-631, 1989.

28. Jensen RA, Page DL and Holt JT: Identification of genes expressed in premalignant breast disease by microscopy-directed cloning. Proc Natl Acad Sci USA 91: 9257-9261, 1994.

29. Takeda $E$ and Weber G: Role of ribonucleotide reductase in expression of the neoplastic program. Life Sci 28: 1007-1014, 1981.

30. Krieg AM: CpG motifs in bacterial DNA and their immune effects. Annu Rev Immunol 20: 709-760, 2002.

31. Miraglia L, Watt AT, Graham MJ and Crooke ST: Variations in mRNA content have no effect on the potency of antisense oligonucleotide. Antisense Nucleic Acid Drug Dev 10: 453-461, 2000 . 